\title{
How personal standards perfectionism and evaluative concerns perfectionism affect the error positivity and post-error behavior with varying stimulus visibility
}

\author{
Jessica Drizinsky ${ }^{1,2}$ • Joachim Zülch ${ }^{1} \cdot$ Henning Gibbons ${ }^{3}$ - Jutta Stahl ${ }^{2}$
}

Published online: 1 June 2016

(C) Psychonomic Society, Inc. 2016

\begin{abstract}
Error detection is required in order to correct or avoid imperfect behavior. Although error detection is beneficial for some people, for others it might be disturbing. We investigated Gaudreau and Thompson's (Personality and Individual Differences, 48, 532-537, 2010) model, which combines personal standards perfectionism (PSP) and evaluative concerns perfectionism (ECP). In our electrophysiological study, 43 participants performed a combination of a modified Simon task, an error awareness paradigm, and a masking task with a variation of stimulus onset asynchrony ( $\mathrm{SOA} ; 33$, 67 , and $100 \mathrm{~ms}$ ). Interestingly, relative to low-ECP participants, high-ECP participants showed a better post-error accuracy (despite a worse classification accuracy) in the highvisibility SOA 100 condition than in the two low-visibility conditions (SOA 33 and SOA 67). Regarding the electrophysiological results, first, we found a positive correlation between ECP and the amplitude of the error positivity (Pe) under conditions of low stimulus visibility. Second, under the condition of high stimulus visibility, we observed a higher Pe amplitude for high-ECP-low-PSP participants than for high-ECP-highPSP participants. These findings are discussed within the framework of the error-processing avoidance hypothesis of
\end{abstract}

Electronic supplementary material The online version of this article (doi:10.3758/s13415-016-0438-z) contains supplementary material, which is available to authorized users.

Jessica Drizinsky

Jessica.Drizinsky@ise.ruhr-uni-bochum.de

1 Chair of Industrial Sales Engineering, Ruhr-University Bochum, Bochum, Germany

2 Department of Psychology, University of Cologne, Cologne, Germany

3 Department of Psychology, University of Bonn, Bonn, Germany perfectionism (Stahl, Acharki, Kresimon, Völler, \& Gibbons, International Journal of Psychophysiology, 97, 153-162, 2015).

Keywords Error positivity $\cdot$ Event-related potentials $\cdot$ Error detection $\cdot$ Personal standards perfectionism $\cdot$ Evaluative concerns perfectionism

The detection of erroneous performance is an important cognitive mechanism that allows an individual to correct an error immediately or to avoid incorrect behavior in the future. Even if the consequences of imperfect behavior are not severe, the awareness of an error can be annoying for some people, because it could be a demonstration of one's own imperfection. Perfectionists are people with a low error tolerance, and the perfectionism model of Gaudreau and Thompson (2010, 2011; Gaudreau, 2013) comprises two interacting subtraits of perfectionism: personal standards perfectionism (PSP) and evaluative concerns perfectionism (ECP). High PSP describes stable characteristics of the individual's (intrinsic) motivation to show an excellent performance and high goal orientation, which is based on high criteria for good performance (Frost, Marten, Lahart, \& Rosenblate, 1990), whereby these internal criteria are not necessarily related to the standards of others (Hewitt \& Flett, 1991). High ECP refers to a stable characteristic associated with the individual's emotional responses, such as the concern about being poorly evaluated by others because of one's own imperfect behavior (e.g., Frost et al., 1990). The anticipated standards of others affect the attitude of individuals with high ECP toward erroneous behavior, which means that ECP reflects an other-oriented type of perfectionism (Hewitt \& Flett, 1991). Gaudreau and Thompson's (2010) model allows for a dimensional as well as a group-based approach, because they suggested four 
subtypes of perfectionism that result from the combination of the two dimensions: nonperfectionist (low ECP, low PSP), pure ECP (high ECP, low PSP), pure PSP (low ECP, high PSP), and mixed perfectionist (high ECP, high PSP). In healthy samples (Stahl, Acharki, Kresimon, Völler, \& Gibbons, 2015; Tops, Koole, \& Wijers, 2013) as well as clinical samples (Pieters et al., 2007; Schrijvers, De Bruijn, Destoop, Hulstijn, \& Sabbe, 2010), variations have been observed in the neural correlates of error processing as a function of perfectionism.

\section{Neural correlates of error awareness}

For different reasons, error awareness is an important aspect for perfectionists. A neural indicator of error awareness, the error positivity ( $\mathrm{Pe}$; Falkenstein, Hohnsbein, Hoormann, \& Blanke, 1991), is a positive component of the event-related potential (ERP) that occurs around $300 \mathrm{~ms}$ after an erroneous response with a centro-parietal scalp distribution. Various theories regarding the functional significance of the Pe have been established, such as the affective-processing theory (e.g., Falkenstein, Hoormann, Christ, \& Hohnsbein, 2000) and the behavior-adaptation theory (e.g., Overbeek, Nieuwenhuis, \& Ridderinkhof, 2005; see also Danielmeier \& Ullsperger, 2011; Schroder \& Infantolino, 2013). Most important for the present approach is the error-awareness theory of the Pe, which is widespread and premised on results showing a higher Pe amplitude for detected than for undetected errors (e.g., Nieuwenhuis, Ridderinkhof, Blom, Band, \& Kok, 2001; O'Connell et al., 2007; Shalgi, Barkan, \& Deouell, 2009). Therefore, it is assumed that the Pe reflects error awareness, and furthermore that the size of the Pe amplitude represents the accumulated decision evidence (Steinhauser \& Yeung, 2010, 2012; for further discussion, see also Bode \& Stahl, 2014) that is required to decide whether a given response was correct or incorrect.

\section{Objective of the study}

As we noted above, some electrophysiological studies have shown perfectionism-related differences in error processing. A positive relationship between ECP and the Pe amplitude (e.g., Schrijvers et al., 2010; Tops et al., 2013) could be a sign of higher affective involvement after error commission among high-ECP participants. Stahl et al. (2015) found both higher Pe amplitudes and higher post-error accuracy for high-PSP relative to low-PSP participants (i.e., a higher percentage of correct responses after error responses), which points to faster error evidence accumulation being used to improve performance after error commission. This finding has raised the questions of whether high-PSP participants show better error detection performance than low-PSP participants, and whether this is related to the Pe variations. Although Stahl and colleagues did not reveal any significant ECP-Pe relationships, they demonstrated a reduced post-error accuracy for highECP participants, which is in line with the authors' errorprocessing avoidance hypothesis. In their study, pure-ECP participants (high ECP, low PSP) had reduced early errorprocessing activity (as indicated by the error negativity; for details, see Stahl et al., 2015), which was explained by pureECP participants' difficulty with shifting, or inability to shift, attention from task-unrelated to task-related cognitions (Moser, Moran, Schroder, Donnellan, \& Yeung, 2013). This could be a result of worrying about being negatively evaluated (high ECP), yet simultaneously having low intrinsic motivation for performance improvement (low PSP).

The present paradigm was specifically designed to examine perfectionism-related differences in error awareness and posterror behavior. We combined a modified Simon task (Simon, 1969), a masking task (Gibbons, Fritzsche, Bienert, Armbrecht, \& Stahl, 2011; see also Bode et al., 2012), and an error awareness paradigm (e.g., Nieuwenhuis et al., 2001). Thereby, we aimed at varying both the response uncertainty (induced by low visibility) and the error awareness by experimentally varying the visibility of the target stimuli. In general, we expected to find a greater number of correct responses, faster responses, more correct decisions (i.e., errors being classified as errors and correct responses being classified as correct responses), variation in post-response behavior (accuracy and response times), and higher Pe amplitudes in error trials with increasing visibility of the stimuli (Gibbons et al., 2011).

On the basis of prior perfectionism-related findings (Schrijvers et al., 2010; Stahl et al., 2015; Tops et al., 2013), we expected to find a higher Pe amplitude for participants with high PSP than for those with low PSP. If this is a sign of PSPrelated variations in error-processing efficiency, it should be reflected in a greater number of correct decisions and a higher post-error accuracy (perhaps accompanied by post-error slowing) for high-PSP than for low-PSP participants. Because ECP seems to affect task-specific attention (Moser et al., 2013) and emotional aspects of error processing (e.g., Stahl et al., 2015), we examined the moderating effects of ECP on the relationships between PSP and error correlates.

\section{Method}

\section{Participants}

Forty-three students (21 females, 22 males) from the University of Cologne, ranging in age from 19 to 41 years (mean \pm standard deviation: $25.5 \pm 5.0$ years), participated in the present study. They were either paid ( $€ 8.00 / \mathrm{h})$ or received course credits for their participation. All participants 
reported normal or corrected-to-normal vision, and none of them declared a neurological or a psychiatric disorder. Six of the participants $(14.6 \%)$ were left-handed. Two had to be excluded because of technical artifacts in the electrophysiological recordings. Thus, the data set for further analysis consisted of 41 (19 female, 22 males) participants. Prior to the investigation, informed written consent was given, and the study was approved by the ethics committee of the German Psychological Association.

\section{Psychometric assessment}

The German version of the Frost Multidimensional Perfectionism Scale (FMPS) by Frost et al. (1990; AltstötterGleich \& Bergemann, 2006) was used to assess ECP, using the Concern over Mistakes scale (nine items), and PSP, using the Personal Standards scale (seven items). The item scores ranged from 1 (strongly disagree) to 5 (strongly agree). The participants' scores were computed as the mean scores. Therefore, for both scales the minimum score was 1 and the maximum score was 5 . Missing values were replaced with the average scale value of the participant.

\section{Stimuli and procedure}

The imperative stimulus was a white arrowhead on a black background pointing to the left or the right ("<" or " >”; horizontal visual angle $=0.33^{\circ}$ ). A white fixation cross ("+"”; horizontal visual angle $=0.31^{\circ}$ ) was presented in the center of the screen. The arrow was presented in the center of one of two white square frames placed to the left and right of the center (horizontal angle $4.2^{\circ}$, vertical angle $1.2^{\circ}$ ) on a black background. As in a Simon task (Simon, 1969), congruent and incongruent trials were presented. In congruent trials, the direction of the arrow coincided with the position (left or right) of the square frame, whereas in incongruent trials, the direction of the arrow did not coincide with the frame's position.

Before the onset of the imperative stimulus, white-colored dot masks (white-noise distributed, 117-ms duration) were presented in both square frames; the arrow was visible for 100,67 , or $33 \mathrm{~ms}$ (stimulus onset asynchrony [SOA]), and was followed by a second mask (117-ms duration). The SOA conditions were presented blockwise (two blocks for each SOA), because a mixed presentation decreased the chance of finding SOA effects on the ERP components (see Gibbons et al., 2011, Exp. 1). The order of the SOA blocks was randomized across participants, who were instructed to press as quickly as possible the key (left or right) that corresponded to the direction of the arrow. If the keys were not pressed within an individually adapted interval of time, the white square frames turned red, and the trial was excluded from further analyses. The response interval was calculated as $90 \%$ of the mean response time (RT) of the practice block.
If participants responded in time, a white question mark $(0.5 \mathrm{~mm})$ replaced the fixation cross $1,588 \mathrm{~ms}$ after the imperative stimulus onset. The participant was asked to press one of the two keys if she or he was certain that the response was incorrect (second-response interval of 1,732 ms after onset of the question mark). The use of these very specific durations had no task-inherent reasons. The experiment comprised six experimental blocks and two practice blocks at the beginning of each session; each block contained 72 trials.

\section{Apparatus}

The stimuli were presented on a 17 -in. SVGA monitor (refresh rate: $60 \mathrm{~Hz}$ ). To ensure equal distances to the monitor and to reduce head movements, a chin rest was fixed $59 \mathrm{~cm}$ from the monitor. The behavioral data were assessed by forcesensitive keys. The keys comprised plastic cuboids, which were attached to flexible steel plates. The ends of the plates were fixed by metal clamps with strain gauges, measuring the flexion of the index finger with a $500-\mathrm{Hz}$ sampling rate. Both keys were fixed to wooden boards on the right and left sides of the monitor, in a position that was comfortable to the participants.

\section{Behavioral data}

The percentage of errors was computed as the percentage of errors among all responses. For error trials, we further determined the percentage of errors classified as correct (i.e., the percentage of errors classified as correct among all errors). ${ }^{1}$ We also used the signal detection theory approach (Green \& Swets, 1974), but as none of the parameters (e.g., sensitivity and response bias) showed any relationships with one of the perfectionism scores, so for the sake of brevity, we do not report the results in more detail. The classification accuracythat is, the rate of correct decisions - was determined by dividing the number of correct decisions (i.e., errors classified as errors and correct responses classified as correct responses) by the number of all decision constellations. Wrong decisions were defined as errors classified as correct and correct responses classified as errors.

The $R T$ was defined as the interval of time from the imperative stimulus's onset to the first point in time at which a response force of 50 centinewtons was exceeded. Postresponse behavior was assessed by the post-response slowing and post-response accuracy (e.g., Schroder \& Infantolino, 2013). Post-response slowing after an error trial (i.e., posterror slowing) was defined as the difference between the mean

\footnotetext{
${ }^{1}$ We do not use the terms "aware error" and "unaware error" that are normally used, because one cannot rule out that the decision itself was incorrect. Thus, we use the more technical terms "errors classified as correct" (unaware) and "errors classified as incorrect" (aware).
} 
RT in trials following an error and the mean RT in trials preceding an error (i.e., $\mathrm{MRT}_{\mathrm{E}+1}-\mathrm{MRT}_{\mathrm{E}-1}$ ). Dutilh et al. (2012) suggested this more robust measure, as compared to the traditional ones (cf. Rabbitt, 1966), because it accounts for the confounding effects caused by differences in the distribution of errors across time in an experimental session. Analogously, we determined the post-response slowing after correctresponse trials (i.e., post-correct slowing) so as to compare any differences between the two response types. Furthermore, to compare our results with previous studies and account for variations in pre-error RTs, we also used the traditional approach and calculated the post-correct RT (the mean RT on trials following a correct response) and post-error RT (the mean RT on trials following an erroneous response).

The post-response accuracy after error trials (i.e., posterror accuracy) was defined as the percentage of correct responses in trials following an error (i.e., the number of correct responses after an error divided by the total number of errors, multiplied by 100; e.g., $20 \%$ errors after an error, and consequently $80 \%$ correct responses after error commissions, would mean $80 \%$ post-error accuracy). Analogously, the post-response accuracy after correct responses (post-correct accuracy) was defined as the percentage of correct responses in trials following a correct response.

\section{Electrophysiological recordings}

The electroencephalogram (EEG) was recorded with $60 \mathrm{Ag}-$ AgCl electrodes (Fp1, Fp2, AF7, AF3, AFz, AF4, AF8, F7, F5, F3, F1, Fz, F2, F4, F6, F8, FT7, FC5, FC3, FC1, FCz, FC2, FC4, FC6, FT8, T7, C5, C3, C1, Cz, C2, C4, C6, T8, TP7, CP5, CP3, CP1, CPz, CP2, CP4, CP6, TP8, P7, P5, P3, P1, Pz, P2, P4, P6, P8, PO7, PO3, POz, PO4, PO8, O1, Oz, and $\mathrm{O} 2$ ). The electrodes were referenced against the left mastoid. The vertical electrooculogram (EOG) was monitored from electrodes positioned infraorbitally to the left eye, and the bipolar horizontal EOG was recorded from two electrodes placed $2 \mathrm{~cm}$ laterally from the outer canthi. The EEG was recorded continuously at a sampling rate of $500 \mathrm{~Hz}$ using a BrainAmp DC (Brain Products Ltd., Munich, Germany) amplifier. The time constant was infinite. For all channels, the low-pass filter was set to $70 \mathrm{~Hz}$. BrainVision Software 2.02 (Brain Products) was used for the data analysis. Responselocked ERPs were analyzed, with intervals ranging from $100 \mathrm{~ms}$ before until $500 \mathrm{~ms}$ after the response to the imperative stimulus. The 100-ms interval before the response onset served as the baseline. The ocular correction algorithm of Gratton, Coles, and Donchin (1983) was used for eye movement correction, if required. The automatic artifact rejection excluded all trials with amplitudes above/below $\pm 150 \mu \mathrm{V}$. To prevent an overlap of simultaneous activity of contiguous electrodes, current-source density (CSD; Perrin, Pernier, Bertrand, \& Echallier, 1989) transformations were implemented (e.g., Gibbons et al., 2011; Vidal, Burle, Bonnet, Grapperon, \& Hasbroucq, 2003; Vidal, Hasbroucq, Grapperon, \& Bonnet, 2000). The order of the splines was 4, the maximal degree of the Legendre polynomial was 10 , and the $\lambda$ regularization value was $1.000000 \mathrm{e}-005$.

\section{Electrophysiological data}

As dependent electrophysiological measures, the amplitudes and latencies of the $\mathrm{Pe}$ (i.e., the positivity in error trials) and the Pc (i.e., the positivity in correct-response trials) were determined from the individual mean CSD-ERP waveforms, separately for each combination of $\operatorname{SOA}(33,67,100)$, Response Type (correct response, error), and Decision (correct decision, wrong decision). The time window and maximum of the Pe/Pc vary across studies (e.g., Overbeek et al., 2005). In our study, the Pe/Pc amplitude was defined as the most positive mean amplitude for the CSD-ERP waveforms within a time window from 200 to $350 \mathrm{~ms}$ after the response at $\mathrm{Cz}$, where the component was most prominent (see also, e.g., Herrmann, Römmler, Ehlis, Heidrich, \& Fallgatter, 2004). The latency of the $\mathrm{Pe} / \mathrm{Pc}$ was defined as the period of time between the response onset and the peak amplitude.

\section{Statistical analyses}

General linear models (GLMs) were performed for RTs, Pe/Pc amplitudes, and $\mathrm{Pe} / \mathrm{Pc}$ latencies, comprising the withinsubjects factors SOA (33, 67, $100 \mathrm{~ms})$, Response Type (correct response, error), and Decision (correct decision, wrong decision). For pos-tresponse (error and correct) slowing and post-response (error and correct) accuracy, GLMs with the within-subjects factors $\operatorname{SOA}(33,67,100 \mathrm{~ms})$ and Decision (correct decision, wrong decision) were analyzed. A GLM with the within-subjects factor $\operatorname{SOA}(33,67,100 \mathrm{~ms})$ was performed for percentages of errors, percentages of errors classified as correct, and classification accuracy. Since the number of errors in congruent trials was too small to allow us to perform a reliable analysis, the Congruency was not included as a factor in our main analyses (but see Supplement 1).

For the perfectionism-related analyses, the GLMs for RTs, $\mathrm{Pe} / \mathrm{Pc}$ amplitudes and latencies, post-response slowing, postresponse accuracy, classification accuracy, percentages of errors, and percentages of errors classified as correct included the same within-subjects factors as the general analyses, plus the centralized perfectionism scores (PSP, ECP) as continuous predictors. Furthermore, for Pe/Pc amplitudes, GLMs with Response Type (correct response, error) and Decision (correct decision, wrong decision) as within-subjects factors and with centralized perfectionism scores (PSP, ECP) as continuous predictors were performed for every SOA level separately. Within-subjects effects were analyzed using Tukey's HSD 
post-hoc tests, whereas between-subjects effects for the continuous variables were tested by means of moderated regression analyses and simple slope analyses. Greenhouse-Geisser adjustments were applied, if required.

\section{Results}

\section{Psychometric data}

The mean \pm standard deviation of the ECP scores was $2.39 \pm$ 0.77 (ranging from 1 to 4.33 ; centralized scores: -1.40 and 1.94), and that of the PSP scores was $3.25 \pm 0.68$ (ranging from 2.0 to 4.57 ; centralized scores: -1.25 and 1.32). The correlation between the ECP and PSP scores was $\mathrm{r}=.54(p$ $<.001)$.

\section{Behavioral data}

Percentage of errors The GLM on the percentages of errors (independent of the decision) showed a significant main effect of SOA, $F(2,80)=89.7, p<.001, \eta^{2}=.69$. Post-hoc tests showed significant differences only between SOA 33 (34.6\% $\pm 1.6 \%$ ) and the other two SOA conditions (SOA 67: $19.0 \%$ $\pm 1.9 \%$; SOA $100: 15.5 \% \pm 1.8 \%$; both $p \mathrm{~s}<.001$ ). The GLM for the percentage of errors classified as correct showed no significant main effect of SOA $(p>.10)$.

Error types and perfectionism The GLM on the percentages of all errors did not show any relationship with PSP and ECP (all $p \mathrm{~s}>.10$ ). The second GLM, for the percentage of errors classified as correct, including the two perfectionism scores as continuous predictors, yielded a significant effect of PSP, $F(1$, $37)=4.4, p<.05, \eta^{2}=.11$. The post-hoc regression analysis revealed a positive relationship between the percentage of errors classified as correct and the PSP score $(\beta=0.41, p<$ $.05)$, indicating that after error commission, high-PSP participants decided more often that they had responded correctly than did participants with low PSP. No further effect was shown (all $p s>.10)$.

Classification accuracy A GLM performed for the classification accuracy rates differed significantly with $\operatorname{SOA}, F(2,80)$ $=11.9, p<.001, \eta^{2}=.23$. Post-hoc tests showed that the classification accuracy rate for SOA $100(68.2 \% \pm 2.0 \%)$ was significantly higher than those for SOA $67(58.2 \% \pm$ $2.5 \% ; p<.01)$ and SOA $33(55.6 \% \pm 2.4 \% ; p<.001)$, which did not differ $(p>.10)$.

Classification accuracy and perfectionism The GLM that included PSP, ECP, and SOA showed a significant interaction between SOA and ECP, $F(2,74)=3.33, p<.05, \eta^{2}=.08$. This interaction can be explained by the post-hoc regression analyses of the differences in classification accuracy between the SOA conditions, which showed that the interaction resulted from the difference between SOA 100 and SOA $67(\beta=-0.47$, $p<.05$; no other difference showed a significant relationship, all $p \mathrm{~s}>.07)$. In the SOA 67 condition, higher-ECP participants made more correct decisions than did participants with lower $\mathrm{ECP}$, whereas in the SOA 100 condition, participants with higher ECP made fewer correct decisions than did low-ECP participants. No further effect was obtained (all $p \mathrm{~s}>.10$ ).

Response times For mean RTs, we obtained a significant main effect of Response Type, $F(1,26)=10.26, p<.01, \eta^{2}=.28$. Errors were slower $(353 \mathrm{~ms} \pm 23.7 \mathrm{~ms})$ than correct responses (301 $\mathrm{ms} \pm 16.1 \mathrm{~ms})$. Moreover, a significant main effect of Decision, $F(1,26)=10.48, p<.01, \eta^{2}=.29$, showed that responses with correct decisions (339 $\mathrm{ms} \pm 19.4 \mathrm{~ms})$ were slower than responses with wrong decisions $(314 \mathrm{~ms} \pm$ $18.5 \mathrm{~ms})$. The significant interaction between SOA and Response Type, $F(2,52)=7.91, p<.01, \eta^{2}=.23$, and the post-hoc tests revealed a significant difference between errors $(385 \mathrm{~ms} \pm 33.0 \mathrm{~ms})$ and correct responses $(280 \mathrm{~ms} \pm 15.0 \mathrm{~ms})$ only for SOA $100(p<.001)$, whereas no further differences were found for the SOA 67 condition (errors: $367 \mathrm{~ms} \pm 29.6 \mathrm{~ms}$; correct responses: $310 \mathrm{~ms} \pm 17.2 \mathrm{~ms} ; p=.058$ ) and the SOA 33 condition (errors: $307 \mathrm{~ms} \pm 20.4 \mathrm{~ms}$; correct responses: $312 \mathrm{~ms}$ $\pm 17.4 \mathrm{~ms} ; p>.10)$. A significant interaction between Response Type and Decision, $F(1,26)=22.01, p<.001, \eta^{2}=.46$, demonstrated that error responses were significantly slower when participants made a correct decision $(389 \mathrm{~ms} \pm 25.5 \mathrm{~ms})$ than when they made a wrong decision $(318 \mathrm{~ms} \pm 24.7 \mathrm{~ms} ; p<$ $.001)$. No RT differences were observed for correct responses (correct decision: $290 \mathrm{~ms} \pm 17.5 \mathrm{~ms}$; wrong decision: $311 \mathrm{~ms} \pm$ $15.3 \mathrm{~ms} ; p>$.10). Finally, we observed a significant three-way interaction of SOA, Response Type, and Decision, $F(2,52)=$ $4.03, p<.05, \eta^{2}=.13$. Longer RTs for the two types of error responses (correct and wrong decisions) than for the two correct responses (correct and wrong decisions) were found in the SOA 100 condition (all $p$ s $<.05$ ). In the SOA 67 condition, only the correctly classified error responses were significantly slower than the other three response types. No further significant effects were obtained. A congruency effect on RTs was also investigated (see Supplement 1).

RTs and perfectionism The GLM performed on mean RTs, which included SOA, Response Type, and Decision as withinsubjects factors and PSP and ECP as continuous variables, could not provide any significant perfectionism-related effects on RTs.

Post-response behavior A GLM on the post-response slowing for error trials and correct trials (Dutilh et al., 2012), including Response Type, SOA, and Decision as factors, revealed an effect of Decision, $F(1,26)=9.53, p<.001, \eta^{2}=.38$, showing 
post-response slowing after responses that were classified as errors $(47.4 \mathrm{~ms} \pm 15.6 \mathrm{~ms})$, whereas no post-response slowing occurred after responses classified as correct $(-3.7 \mathrm{~ms} \pm$ $4.3 \mathrm{~ms})$. A significant Response Type $\times$ SOA interaction also emerged, $F(1,26)=3.19, p<.05, \eta^{2}=.11$. The post-hoc test demonstrated a significant difference between post-error slowing and post-correct slowing only in the SOA 100 condition (error: $69.2 \mathrm{~ms} \pm 29.3 \mathrm{~ms}$; correct: $0.0 \mathrm{~ms} \pm 8.3 \mathrm{~ms}$ ). No further effects were obtained (all $p \mathrm{~s}<.10$ ). The traditional postresponse-slowing measures came to the same results-for the sake of brevity, they are not reported in detail. The GLM on post-response accuracy (Response Type, SOA, and Decision) showed a significant effect of SOA, $F(2,52)=34.70, p<.001$, $\eta^{2}=.57$. The percentage of post-response errors was higher for the SOA 33 condition $(42.5 \% \pm 1.91 \%)$ than either for the SOA 66 condition $(27.4 \% \pm 2.15 \% ; p<.001)$ or for SOA 100 $(23.9 \% \pm 2.50 \% ; p<.001$; we observed no significant difference between SOA 66 and SOA 100, $p>.10$ ) condition. No further effect was obtained (all $p \mathbf{s}<.10$ ). This result is similar to that for the general error rates, which were smaller for longer SOAs.

Post-response behavior and perfectionism No significant main effect or interaction of PSP and ECP with SOA and Decision was obtained for post-response slowing scores (i.e., error and correct; all $p \mathrm{~s}>.10$ ). The GLM of post-response accuracy (i.e., error and correct) showed no effect of PSP or ECP on post-correct accuracy, whereas the analyses of posterror accuracy yielded a significant interaction between ECP and SOA, $F(2,74)=4.0, p<.05, \eta^{2}=.14$. The post-hoc regression analyses showed a significant positive relationship between ECP and post-error accuracy in the SOA 100 condition (SOA 100: $\beta=0.54, p<.05$ ), but not in the two shorter SOA conditions (all $p \mathrm{~s}>.10$ ), indicating a performance improvement after error commission for participants with higher $\mathrm{ECP}$, as compared to low-ECP participants, only in the condition with high visibility.

\section{Electrophysiological data}

$\mathbf{P e} / \mathbf{P c}$ component In Fig. 1, the ERP waveforms are depicted time-locked to the response onset as a function of Response Type, Decision, and SOA. First, we found a significant main effect of Response Type, $F(1,25)=15.92, p<.001, \eta^{2}=.39$. The Pe amplitude after errors $\left(0.233 \pm 0.033 \mu \mathrm{V} / \mathrm{cm}^{2}\right)$ was larger than that after correct responses $(0.125 \pm 0.026 \mu \mathrm{V} /$ $\mathrm{cm}^{2}$ ). In addition, a significant SOA $\times$ Response Type interaction was revealed, $F(2,50)=6.33, p<.01, \eta^{2}=.20$. The Pe/ $\mathrm{Pc}$ amplitude differences between errors and correct responses differed significantly in the two longer SOA conditions (Fig. 2A, all $p s<.001$ ) than in the short SOA condition, in which no significant difference was found $(p>.10)$. Furthermore, a significant interaction between Response

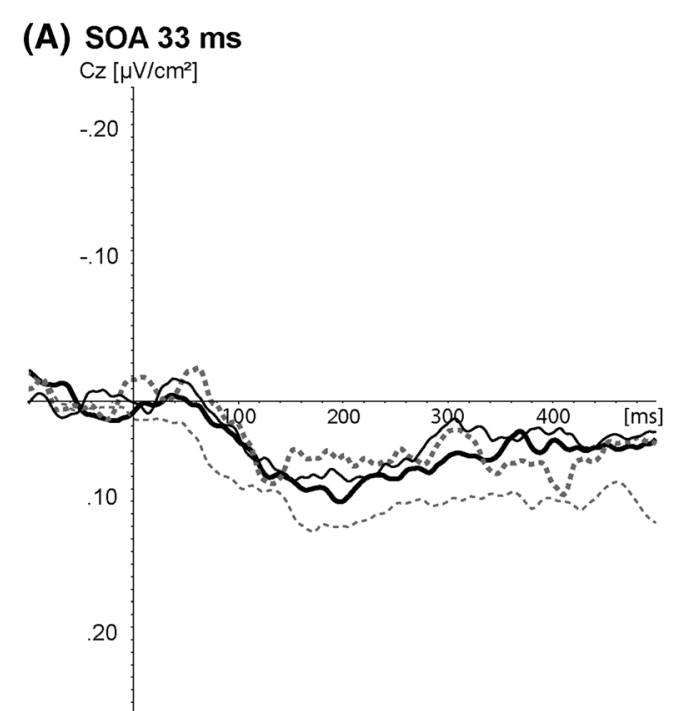

(B) SOA $67 \mathrm{~ms}$

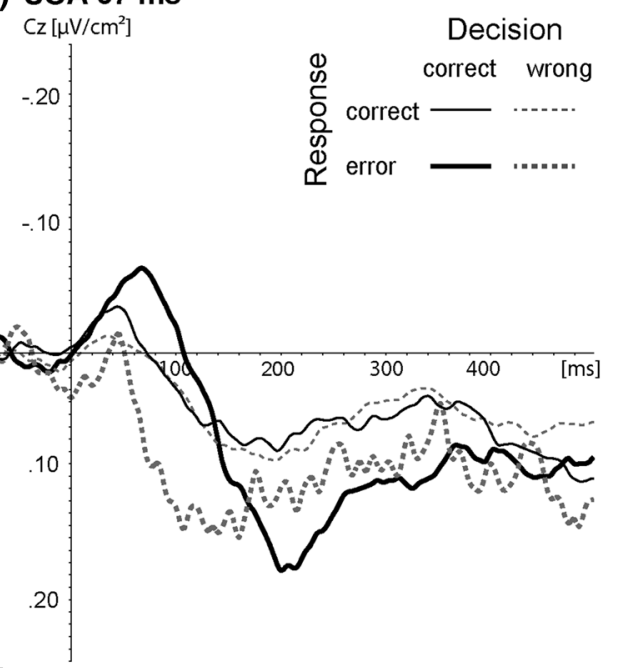

(C) SOA $100 \mathrm{~ms}$

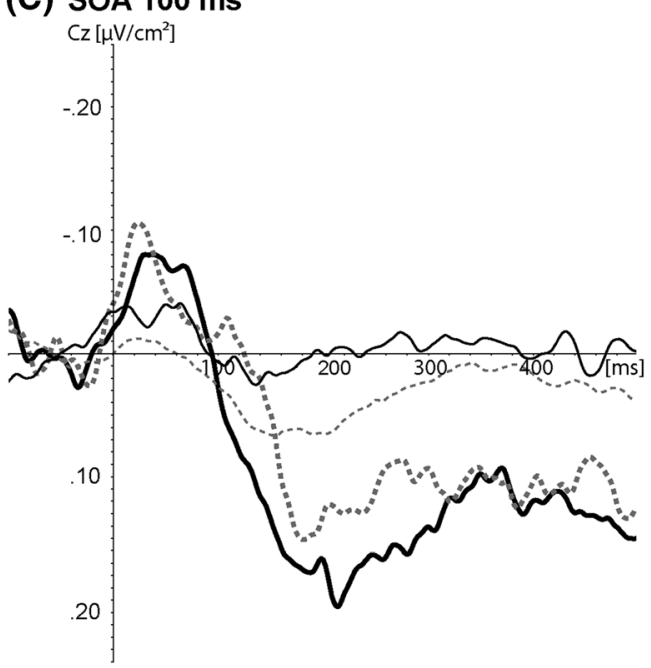

Fig. 1 Current-source density (CSD) transformed event-related potential waveforms time-locked to the response onset $(0 \mathrm{~ms})$ as a function of Response Type and Decision at electrode Cz. Waveforms are shown separately for stimulus onset asynchrony (SOA) 33 (A), 67 (B), and $100(\mathrm{C})$ 


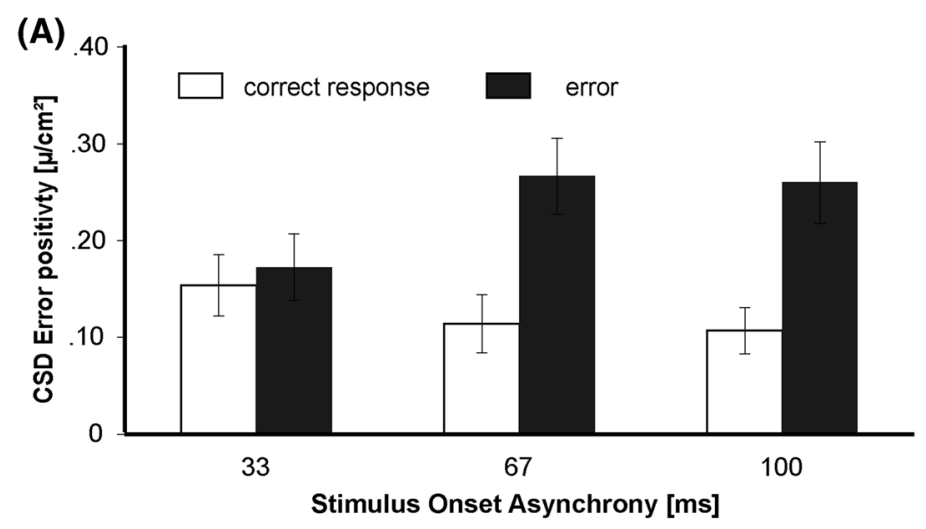

(B)

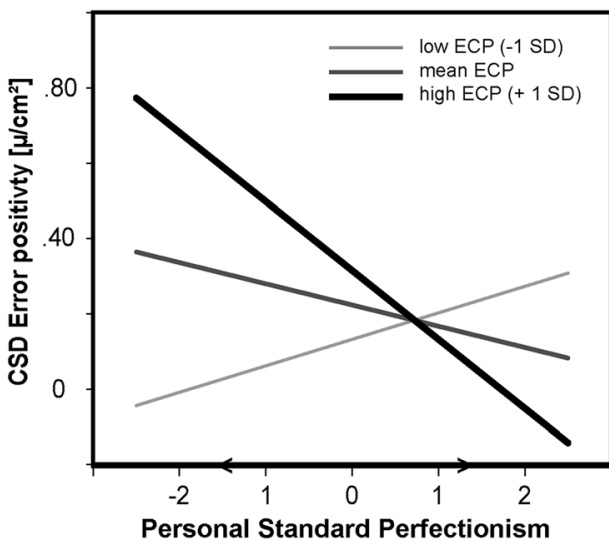

(C)

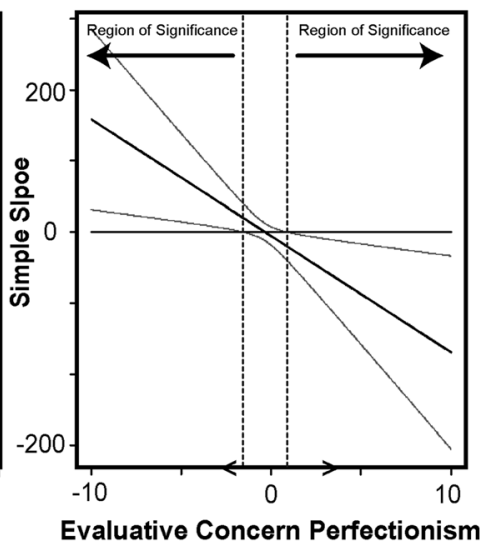

Fig. 2 (A) Current-source density (CSD) transformed Pe/Pc amplitude, as a function of stimulus onset asynchrony (SOA) and Response Type. (B) Results of the simple slope analysis of the interaction between personal standards perfectionism and evaluative concerns perfectionism for mean $\mathrm{Pe} / \mathrm{Pc}$ amplitude in the SOA 100 condition. (C) Simple slopes for ECP, and the confidence intervals. The region of significance is defined by a confidence range not including zero. The ranges of the observed centralized ECP scores and centralized PSP scores in our sample are indicated by the arrowheads

responses). No significant relationship was apparent in the SOA 67 condition (all $p \mathrm{~s}<.05$ ).

Furthermore, the GLM for the SOA 100 condition showed a significant interaction between PSP and ECP, $F(1,25)=4.6, p<$ $.05, \eta^{2}=.16$. Figure $3 \mathrm{~B}$ shows the ERP waveforms time-locked to the response onset as a function of ECP and PSP for SOA 100 (see also Supplement 3, Figs. S1C and S1D, for separate illustrations of errors and correct responses). A simple slope analysis showed a significant difference in slopes, $t(41)=$ $-2.09, p<.05$. Figure 2B illustrates the varying regressionbased slopes for the relationship between PSP and mean $\mathrm{Pe} / \mathrm{Pc}$ amplitude as a function of ECP. A significantly negative slope of the PSP-Pe/Pc relationship was shown with higher ESP scores (+1 standard deviation), whereas a significantly positive slope was obtained for low ECP scores ( -1 standard deviation). The slope was close to zero for the mean ECP scores (mean of the centralized scores $=0$ ). The region of significance for the slopes (Fig. 2C) indicates that ECP scores below the mean (scores below -1.5 ) or above the mean (scores above +0.8 ) significantly moderated the slopes of the relationship between 


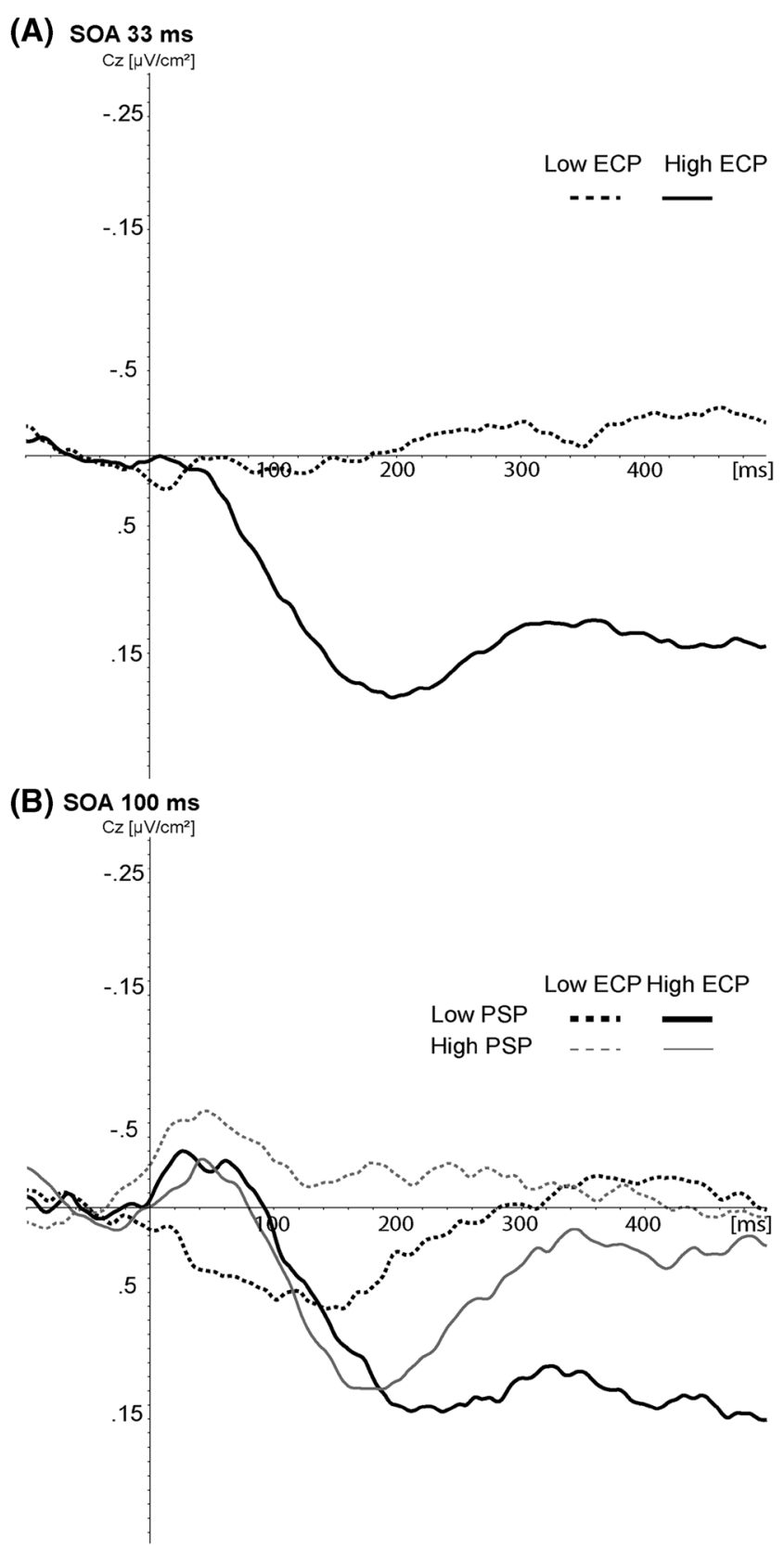

Fig. 3 Current-source density transformed event-related potential waveforms, time-locked to the response onset $(0 \mathrm{~ms})$ at electrode $\mathrm{Cz}$, as a function of ECP for the stimulus onset asynchrony (SOA) of 33 (A), and of both ECP and PSP for the SOA of 100 (B)

PSP and Pe/Pc. No further significant perfectionism-related findings on the $\mathrm{Pe} / \mathrm{Pc}$ amplitude was obtained, even by the use of the overall perfectionism scale (not reported in detail). No effects on $\mathrm{Pe} / \mathrm{Pc}$ latency was obtained (all $p \mathrm{~s}>.10$ ).

\section{Discussion}

Committing errors is part of human nature. Nevertheless, people differ greatly when dealing with imperfect behavior.
Gaudreau and Thompson (2010) distinguished two interacting subtraits, PSP and ECP, reflecting perfectionism-related intrinsic and extrinsic motivational and emotional characteristics. By means of $\mathrm{Pe} / \mathrm{Pc}$ and several behavioral measures, our aim was to investigate differences in error detection that can be explained by variations of the two subtraits, using a combination of a Simon task (Simon, 1969), a masking task (e.g., Gibbons et al., 2011), and an error awareness paradigm (e.g., Nieuwenhuis et al., 2001) to assess variability in error processing.

\section{Behavioral aspects of error processing}

An increasing task difficulty with decreasing stimulus visibility was suggested by both a higher percentage of errors and poorer classification accuracy. Furthermore, for post-response behavior we found a higher percentage of post-response errors with decreasing stimulus visibility, a post-response slowing after responses that were classified as errors, and a significant difference between post-error slowing and post-correct slowing in the SOA 100 condition. These results demonstrated that both error detection and behavioral adaptation after error commission became more likely with high stimulus visibility. In contrast to previous findings, errors were slower than correct responses in SOA 100, indicating that error commission was predominantly not a result of rash, imprecise stimulus processing (e.g., Rabbitt, 1966) but of impeded stimulus processing (see also Gibbons et al., 2011). Although the decision had no effect on the speed of correct responses, the responses were slower for correctly classified errors than for incorrectly classified ones, but only in the SOA 67 condition. The chance of developing an exact stimulus-response representation should be more likely in the SOA 67 condition than in the SOA 33 condition, in which responses should mainly be based on guessing or subliminal stimulus processing (e.g., Bode et al., 2012); however, the chance of developing an aware correct stimulus-response representation in time was presumably not always available. One can assume a race between a fast, guess-based response initiation of the incorrect hand and the inhibition process starting after delayed (successful) stimulus detection. However, if an internal response threshold has been exceeded (the point of no return; Bartlett, 1958), the error cannot be prevented but is nonetheless classified as an error. Likely, such a response-slowing, second inhibitory process was not activated in incorrectly classified errors, because the errors did not reach the level of awareness. Several studies have demonstrated that response inhibition increases RTs (for a review, see Burle, Vidal, Tandonnet, \& Hasbroucq, 2004).

\section{Perfectionism and behavioral aspects of error processing}

The investigation of perfectionism-related variations has shown a positive relationship between the PSP score and the 
percentage of errors classified as correct, indicating that highPSP participants tend to classify an incorrect response more often as a correct response than do low-PSP participants. By definition, people with high PSP have high demands on their own performance (e.g., Frost et al., 1990). The observed decision tendency (to classify errors more often as correct) could be a sign of the high self-esteem of high-PSP participants (e.g., Wang, 2012) or of having trouble with admitting imperfect behavior. The present data do not allow us to distinguish between these two perspectives. Since the relationship was independent of SOA, we postulate that perfectionism-related variations in neither perceptual processing nor error evidence accumulation (cf. Steinhauser \& Yeung, 2010, 2012) account for the present findings. Even in the SOA 33 condition, with a rather low chance of error evidence accumulation, high-PSP participants more often stated that their responses were correct than low-PSP participants did.

The ECP-by-SOA interaction of classification accuracy showed, at first glance, counterintuitive relationships: As compared to low-ECP participants, those with high ECP scores more often decided correctly in the condition with medium visibility (SOA 67: positive relationship), whereas they made fewer correct decisions with high visibility (SOA 100: negative relationship) and no variation with perfectionism was obtained in the SOA 33 condition. The differential effects of stimulus visibility (and the resulting response certainty), however, could account for this. In SOA 33 trials, the participants did not have a chance to establish a reliable stimulus-response representation, and decisions were mainly based on guessing instead of actual error evidence. Thus, evaluative concerns might not be triggered. In the longer SOA conditions, highECP participants are generally not worse at error detection, as was assumed by Stahl et al. (2015). For example, the higher post-error accuracy of high-ECP participants demonstrated better post-error adaptation in the SOA 100 condition. The poorer classification performance in this condition, however, is puzzling. The chance to develop error awareness might have increased task-unrelated cognitions (e.g., general worrying; Moser et al., 2013) in high ECPs, which attenuated the attention to response processing (Stahl et al., 2015) and worsened error awareness. The present results further indicate that post-error accuracy does not necessarily require error awareness. In the SOA 67 trials, the reduced chance of error awareness increased the classification performance of highECP participants. Although their evidence accumulation should have been better compared to SOA 33, several responses and the following decisions should result from guessing. With a weak visibility, error commission could be attributed to external causes, which might have reduced the subjective threat of poor evaluation, and no additional task-unrelated cognitions attenuated attention. Hence, the high-ECP participants were able to show improved classification performance. Importantly, the perfectionism-related performance differences cannot be explained by a shift in the speed-accuracy trade-off. Neither RT nor post-response-slowing effects were found.

\section{Electrophysiological aspects of error processing}

As expected (Gibbons et al., 2011), a Pe/Pc amplitude difference between errors and correct responses was observed in the longer SOA conditions (SOA 67, SOA 100), but not in the SOA 33 condition, which is in line with the error evidence accumulation view (Steinhauser \& Yeung, 2010, 2012). Since the visibility of the stimuli was very poor in the SOA 33 condition, participants often - but not always (the response rate was above chance, $65.4 \%$ ) - had to guess. Therefore, the percentage of errors was higher than in the two longer SOA conditions, and likewise, error evidence accumulation was presumably rather small, and no $\mathrm{Pe} / \mathrm{Pc}$ amplitude difference between errors and correct responses was observed in this SOA condition. Furthermore, the $\mathrm{Pe} / \mathrm{Pc}$ amplitude was supposed to be higher for aware errors than for unaware errors; however, no reliable evidence was obtained. The small number of correct as compared to incorrect decisions might have reduced the signal-to-noise ratio of the ERP component. Alternatively, the assessment of error awareness could have influenced our results (for further discussion, see below).

\section{Perfectionism and electrophysiological aspects of error processing}

A positive relationship between $\mathrm{Pe} / \mathrm{Pc}$ amplitude and ECP scores in a small sample ( $N=17$; Tops et al., 2013) provided the first evidence of higher error evidence accumulation in high-ECP participants, whereas Stahl et al. (2015) did not find any relationship when examining a larger sample $(N=94)$. In the SOA 33 condition, a clear positive relationship between ECP scores and $\mathrm{Pe} / \mathrm{Pc}$ amplitude was shown, whereas no relationship was found in the SOA 67 condition. On the basis of their findings in early (unaware) error processing, Stahl et al. argued that high-ECP participants might tend to avoid error processing because errors are highly aversive events predicting the subjective threat of bad evaluation. In contrast to the SOA 33 condition, the stimulus visibility in Stahl and colleagues' task was always high, and thus the chance of developing certainty about response accuracy and the subjective threat of bad evaluation was high. As we discussed above, this threat might not have developed because no (or insufficient) error evidence was accumulated, due to low stimulus visibility. However, the better the visibility, the higher the chance of actual error evidence accumulation and, consequently, the higher the subjective threat of poor evaluation might have been. Thus, the varying directions of the relationships across SOA conditions could be a sign of the varying attribution styles for imperfect behavior, modulating the attentional focus 
of the error processing of high-ECP participants: from external reasons (e.g., "I blame the low visibility!") to individualinherent reasons (e.g., "I blame my inability!").

In the SOA 100 condition, the relationships between PSP, $\mathrm{ECP}$, and $\mathrm{Pe} / \mathrm{Pc}$ amplitude indicate that with increasing ECP there is a negative relationship between PSP and Pe/Pc amplitude (see Fig. 2B, dark-gray and black lines), whereas with lower ECP scores, the PSP-Pe/Pc relationship tends to be positive (Fig. 2B, light-gray line). Hence, the regression model predicts a high $\mathrm{Pe} / \mathrm{Pc}$ amplitude for pure-ECP perfectionists (high ECP, low PSP). The question is why pure-ECP perfectionists showed the highest $\mathrm{Pe} / \mathrm{Pc}$ amplitude, which is contrary to some of Stahl and colleagues' (2015) assumptions, that (pure) ECP participants avoid error processing by reducing attention to error processing very early. An important difference in the tasks is that we forced the participants to evaluate their responses and, in this way, to focus on their response quality and deal with their imperfections, something that may be more difficult for pure-ECP participants. A performance-monitoring avoidance strategy, however, was not reasonable if they did not want to fail in the decision task. Unfortunately, we observed no evidence in the behavioral data, since there was only an effect of ECP, but PSP did not moderate the effect.

The smaller Pe/Pc amplitude of mixed perfectionists (high PSP, high ECP) might be related to the behavioral finding that high-PSP participants tended to ignore their errors. Remember, they showed a larger number of nondetected errors. The resulting question is which response strategy and cognitive processing were responsible for these findings? This may reflect a rather specific type of error processing avoidance or a very slow error evidence accumulation. To answer this question, further research will be required.

The slightly positive PSP-Pe/Pc relationship of low-ECP participants was in line with the positive $\mathrm{PSP}-\mathrm{Pe} / \mathrm{Pc}$ relation reported by Stahl and colleagues (2015), which was explained in terms of a tendency of high-PSP participants to improve their performance after error commission. However, contrary to the authors' findings, we could not find a positive relationship of PSP scores and post-error behavior. As we discussed above, the additional decision task might have differentially affected the response tendencies. The conscious consideration and evaluation of one's own performance might have had a negative effect on post-error behavior in the present task.

A question that remains is why these $\mathrm{Pe} / \mathrm{Pc}$ findings were shown independently of the actual response outcome (error or correct). This could also be a consequence of the masking task, which made it more difficult to develop the required correct response representation and accumulate error evidence. Note that these findings do not indicate that there is no difference between errors and correct trials, but rather that the difference between $\mathrm{Pe}$ and $\mathrm{Pc}$ is similar for all participants. Thus, the general magnitude of neural activity varies with perfectionism, and the process reflected by the $\mathrm{Pe} / \mathrm{Pc}$ might be activated in most of the trials to evaluate whether or not the given response was correct, which was presumably more important for high perfectionists than for nonperfectionists.

\section{Limitations and further research}

Of course, some potentially relevant aspects were not considered in our study, such as the ERP component peaking immediately before the $\mathrm{Pe} / \mathrm{Pc}$, the so-called error negativity $(\mathrm{Ne} /$ ERN; Falkenstein et al., 1991; Gehring, Goss, Coles, Meyer, \& Donchin, 1993). Whereas some electrophysiological studies (e.g., Pieters et al., 2007; Schrijvers et al., 2010; Stahl et al., 2015; Tops et al., 2013) have shown a relationship between perfectionism and the Ne/ERN, we did not (see Supplement 2), which might be a result of task specifics like the varying stimulus visibility, which might have induced uncertainty, or the difficulty of establishing a stimulus-response representation at an early level of processing. Moreover, we did not examine the influence of response uncertainty in our study, which might have additionally affected the ERP components (see Wessel, 2012). The participants had only two options when evaluating their responses: correct or incorrect. Therefore, we do not know how certain the participants were when they classified their responses. Since the different types of perfectionists presumably differ in their confidence related to performance (e.g., Koivula, Hassmén, \& Fallby, 2002), this could also have differentially affected the neural correlates (the error negativity as well as the error positivity). To investigate the influence of uncertainty more accurately, this binary evaluation could be replaced by confidence ratings in further studies. Another limitation of our study is that our paradigm was not designed to dissociate the different natures of errors. Therefore, we could not distinguish between different types of errors (e.g., perceptual or decisional) in our analysis. Further studies should investigate whether there are perfectionismrelated differences regarding the various natures of errors. Finally, our study included mostly young participants. Other studies have shown that age can have a decreasing effect on Pe/Pc amplitude (e.g., Mathewson, Dywan, \& Segalowitz, 2005). Therefore, age-related variations in perfectionism should also be examined in further research.

\section{Conclusion}

Perfectionism-related variations in behavioral and neurophysiological correlates of error processing were shown. The perceptually driven manipulation of response uncertainty affected response performance, error detection performance, RTs, post-response behavior, and $\mathrm{Pe} / \mathrm{Pc}$ amplitude, but not in the same way for all participants. The perfectionism subtraits PSP and ECP explained some of the observed variance. As 
compared to low-ECP participants, high-ECP participants demonstrated better error awareness with medium visibility, whereas with high-visibility, high-ECP participants demonstrated reduced error awareness but better post-error accuracy. Concurrently, the ECP participants with low PSP showed the higher $\mathrm{Pe} / \mathrm{Pc}$ amplitudes. Taken all together, the $\mathrm{Pe} / \mathrm{Pc}$ amplitude is not necessarily a good predictor of error awareness, and even with reduced error awareness, good post-error accuracy can be shown. The present findings are in line with the error-processing avoidance hypothesis for pure-ECP participants (Stahl et al., 2015), but interestingly, only in a highvisibility condition (evoking low response uncertainty), not in a medium-visibility condition (evoking higher response uncertainty).

Author note We are grateful to Aida Alisic and Jan Gommann for their help with the data acquisition and data analysis. This research was supported by the German Research Foundation, Grant No. STA 1035/3-1.

\section{References}

Altstötter-Gleich, C., \& Bergemann, N. (2006). Testgüte einer deutschsprachigen Version der Mehrdimensionalen Perfektionismus Skala von Frost, Marten, Lahart und Rosenblate (MPS-F). Diagnostica, 52, 105-18. doi:10.1026/0012-1924.52.3.105

Bartlett, F. C. (1958). Thinking: An experimental and social study. New York, NY: Basic Books.

Bode, S., Sewell, D. K., Lilburn, S., Forte, J. D., Smith, P. L., \& Stahl, J. (2012). Predicting perceptual decision biases from early brain activity. Journal of Neuroscience, 32, 12488-98. doi:10.1523/ JNEUROSCI.1708-12.2012

Bode, S., \& Stahl, J. (2014). Predicting errors from patterns of eventrelated potentials preceding an overt response. Biological Psychology, 103, 357-69. doi:10.1016/j.biopsycho.2014.10.002

Burle, B., Vidal, F., Tandonnet, C., \& Hasbroucq, T. (2004). Physiological evidence for response inhibition in choice reaction time tasks. Brain and Cognition, 56, 153-64. doi:10.1016/j.bandc. 2004.06.004

Danielmeier, C., \& Ullsperger, M. (2011). Post-error adjustments. Frontiers in Psychology, 2, 233. doi:10.3389/fpsyg.2011.00233

Dutilh, G., van Ravenzwaaij, D., Nieuwenhuis, S., van der Maas, H. J., Forstmann, B. U., \& Wagenmakers, E.-J. (2012). How to measure post-error slowing: A confound and a simple solution. Journal of Mathematical Psychology, 56, 208-16. doi:10.1016/j.jmp.2012.04. 001

Falkenstein, M., Hohnsbein, J., Hoormann, J., \& Blanke, L. (1991). Effects of crossmodal divided attention on late ERP components: II. Error processing in choice reaction tasks. Electroencephalography and Clinical Neurophysiology, 78, 44755. doi:10.1016/0013-4694(91)90062-9

Falkenstein, M., Hoormann, J., Christ, S., \& Hohnsbein, J. (2000). ERP components on reaction errors and their functional significance: A tutorial. Biological Psychology, 51, 87-107. doi:10.1016/S03010511(99)00031-9

Frost, R. O., Marten, P., Lahart, C., \& Rosenblate, R. (1990). The dimensions of perfectionism. Cognitive Therapy and Research, 14, 44968. doi:10.1007/BF01172967

Gaudreau, P. (2013). The $2 \times 2$ model of perfectionism: Commenting the critical comments and suggestions of Stoeber (2012). Personality and Individual Differences, 55, 351-5. doi:10.1016/j.paid.2013.03. 021

Gaudreau, P., \& Thompson, A. (2010). Testing a $2 \times 2$ model of dispositional perfectionism. Personality and Individual Differences, 48, 532-7. doi:10.1016/j.paid.2009.11.031

Gaudreau, P., \& Thompson, A. (2011). Erratum to "Testing a $2 \times 2$ model of dispositional perfectionism" [Personality and Individual Differences 48 (2010) 532-537]. Personality and Individual Differences, 50, 126. doi:10.1016/j.paid.2010.09.001

Gehring, W. J., Goss, B., Coles, M. G., Meyer, D. E., \& Donchin, E. (1993). A neural system for error detection and compensation. Psychological Science, 4, 385-90. doi:10.1111/j.1467-9280.1993. tb00586.x

Gibbons, H., Fritzsche, A.-S., Bienert, S., Armbrecht, A.-S., \& Stahl, J. (2011). Percept-based and object-based error processing: An experimental dissociation of error-related negativity and error positivity. Clinical Neurophysiology, 122, 299-310. doi:10.1016/j.clinph. 2010.06.031

Gratton, G., Coles, M. G. H., \& Donchin, E. (1983). A new method for off-line removal of ocular artifact. Electroencephalography and Clinical Neurophysiology, 55, 468-84. doi:10.1016/0013-4694(83) 90135-9

Green, D. M., \& Swets, J. A. (1974). Signal detection theory and psychophysics (Revth ed.). Oxford, UK: Krieger.

Herrmann, M. J., Römmler, J., Ehlis, A.-C., Heidrich, A., \& Fallgatter, A. J. (2004). Source localization (LORETA) of the errorrelatednegativity (ERN/Ne) and positivity $(\mathrm{Pe})$. Cognitive Brain Research, 20, 294-9. doi:10.1016/j.cogbrainres.2004.02.013

Hewitt, P. L., \& Flett, G. L. (1991). Perfectionism in the self and social contexts: Conceptualization, assessment, and association with psychopathology. Journal of Personality and Social Psychology, 60, 456-70. doi:10.1037/0022-3514.60.3.456

Koivula, N., Hassmén, P., \& Fallby, J. (2002). Self-esteem and perfectionism in elite athletes: Effects on competitive anxiety and selfconfidence. Personality and Individual Differences, 32, 865-75. doi:10.1016/S0191-8869(01)00092-7

Mathewson, K. J., Dywan, J., \& Segalowitz, S. J. (2005). Brain bases of error-related ERPs as influenced by age and task. Biological Psychology, 70, 88-104. doi:10.1016/j.biopsycho.2004.12.005

Moser, J. S., Moran, T. P., Schroder, H. S., Donnellan, M. B., \& Yeung, N. (2013). On the relationship between anxiety and error monitoring: A meta-analysis and conceptual framework. Frontiers in Human Neuroscience, 7, 466. doi:10.3389/fnhum.2013.0046

Nieuwenhuis, S., Ridderinkhof, K. R., Blom, J., Band, G. P. H., \& Kok, A. (2001). Error-related brain potentials are differentially related to awareness of response errors: Evidence from an antisaccade task. Psychophysiology, 38, 752-60. doi:10. 1017/S0048577201001111

O’Connell, R. G., Dockree, P. M., Bellgrove, M. A., Kelly, S. P., Hester, R., Garavan, H., ... Foxe, J. J. (2007). The role of cingulate cortex in the detection of errors with and without awareness: A high-density electrical mapping study. European Journal of Neuroscience, 25, 2571-9. doi:10.1111/j.1460-9568.2007.05477.x

Overbeek, T. J. M., Nieuwenhuis, S., \& Ridderinkhof, K. R. (2005). Dissociable components of error processing: On the functional significance of the $\mathrm{Pe}$ vis-à-vis the ERN/Ne. Journal of Psychophysiology, 19, 319-29. doi:10.1027/0269-8803.19.4.319

Perrin, F., Pernier, J., Bertrand, O., \& Echallier, J. F. (1989). Spherical splines for scalp potential and current density mapping. Electroencephalography and Clinical Neurophysiology, 72, 184-7. doi:10.1016/0013-4694(89)90180-6

Pieters, G. L. M., de Bruijn, E. R. A., Maas, Y., Hulstijn, W., Vandereycken, W., Peuskens, J., \& Sabbe, B. G. (2007). Action monitoring and perfectionism in anorexia nervosa. Brain and Cognition, 63, 42-50. doi:10.1016/j.bandc.2006.07.009 
Rabbitt, P. M. A. (1966). Errors and error correction in choice-response tasks. Journal of Experimental Psychology, 71, 264-72. doi:10. 1037/h0022853

Schrijvers, D. L., De Bruijn, E. R. A., Destoop, M., Hulstijn, W., \& Sabbe, B. G. C. (2010). The impact of perfectionism and anxiety traits on action monitoring in major depressive disorder. Journal of Neural Transmission, 117, 869-80. doi:10.1007/s00702-010-0419-2

Schroder, H. S., \& Infantolino, Z. P. (2013). Distinguishing between types of errors and adjustments. Journal of Neuroscience, 33, 18356-7. doi:10.1523/JNEUROSCI.4078-13.2013

Shalgi, S., Barkan, I., \& Deouell, L. Y. (2009). On the positive side of error processing: Error-awareness positivity revisited. European Journal of Neuroscience, 29, 1522-32. doi:10.1111/j.1460-9568. 2009.06690.x

Simon, J. R. (1969). Reaction toward the source of stimulation. Journal of Experimental Psychology, 81, 174-6. doi:10.1037/h0027448

Stahl, J., Acharki, M., Kresimon, M., Völler, F., \& Gibbons, H. (2015). Perfect error processing: Perfectionism-related variations in action monitoring and error processing mechanisms. International Journal of Psychophysiology, 97, 153-62. doi:10.1016/j.ijpsycho.2015.06.002

Steinhauser, M., \& Yeung, N. (2010). Decision processes in human performance monitoring. Journal of Neuroscience, 30, 15643-53. doi: 10.1523/JNEUROSCI.1899-10.2010
Steinhauser, M., \& Yeung, N. (2012). Error awareness as evidence accumulation: Effects of speed-accuracy trade-off on error signaling. Frontiers in Human Neuroscience, 6, 240. doi:10.3389/fnhum. 2012.00240

Tops, M., Koole, S. L., \& Wijers, A. A. (2013). The Pe of perfectionism. Concern over mistakes predicts the amplitude of a late frontal error positivity. Journal of Psychophysiology, 27, 84-94. doi:10.1027/ 0269-8803/a000090

Vidal, F., Burle, B., Bonnet, M., Grapperon, J., \& Hasbroucq, T. (2003). Error negativity on correct trials: A reexamination of available data. Biological Psychology, 64, 265-82. doi:10.1016/S0301-0511(03) 00097-8

Vidal, F., Hasbroucq, T., Grapperon, J., \& Bonnet, M. (2000). Is the "error negativity" specific to errors? Biological Psychology, 51, 109-28. doi:10.1016/S0301-0511(99)00032-0

Wang, K. T. (2012). Personal and family perfectionism of Taiwanese college students: Relationships with depression, self-esteem, achievement motivation, and academic grades. International Journal of Psychology, 47, 305-14. doi:10.1080/00207594.2011. 626050

Wessel, J. R. (2012). Error awareness and the error-related negativity: Evaluating the first decade of evidence. Frontiers in Human Neuroscience, 6, 88. doi:10.3389/fnhum.2012.00088 